\title{
Metodología para mejorar la percepción de utilidad de conceptos básicos estadísticos a través de Facebook
}

\begin{abstract}
Ángeles Calduch-Losa ${ }^{a}$, José Antonio Ontalba-Ruipérez ${ }^{\mathrm{b}}$, Enrique Orduna-Malea ${ }^{\mathrm{b}}$, Jorge Serrano-Cobos ${ }^{\mathbf{b}}$, Rosa María Alcover ${ }^{\mathrm{a}}$ y Elena Vázquez ${ }^{\mathrm{a}}$

${ }^{a}$ Departamento de Estadística e Investigación Operativa Aplicadas y Calidad, Universitat Politècnica de València (mcalduch@eio.upv.es, evazquez@eio.upv.es, ralcover@eio.upv.es) y ' Instituto de Diseño y Fabricación (IDF) (joonrui@upv.es, enorma@upv.es, jorserc2@har.upv.es)
\end{abstract}

\begin{abstract}
The main objective of this work is to describe a learning innovation experience aimed to improve the student's perception and understanding about statistical applications in the real world through the use of social networks. The experience was carried out with students of bachelor and master's degrees from the School of Computer Engineering (Universitat Politècnica de València) during the academic course 2015 - 2016. Lecturers involved created a closed Facebook group with the students. By means of the group, teachers shared scientific and informative articles related to the subjects taught, in order to be subsequently commented by the students. Posts (including research on statistical practical cases) were discussed, thereby fostering the participation in a simple, fluent and friendly way by the students.
\end{abstract}

Keywords: Education 2.0, Higher Education, Statistics, Social Networks

\begin{abstract}
Resumen
En este trabajo se presenta una experiencia de innovación docente basada en la utilización de redes sociales. El principal objetivo de la experiencia es que los alumnos mejoren la percepción y comprensión de las aplicaciones de la Estadística en el mundo real. La experiencia fue realizada en el curso 2015 2016 con alumnos de grado y de máster que cursaban sus estudios en la Escuela Técnica Superior de Informática de la Universitat Politècnica de València. Los profesores implicados crearon un grupo cerrado de Facebook a través del cuál compartían artículos científicos y divulgativos relacionados con las asignaturas impartidas, y que comentaban los estudiantes. Estos artículos se discutían fomentando la participación y la investigación de casos prácticos de una forma sencilla, fluida y familiar para el alumno.
\end{abstract}

Palabras clave: Educación 2.0, Educación Superior, Estadística, Redes Sociales 
Metodología para mejorar la percepción de utilidad de conceptos básicos estadísticos a través de Facebook

\section{Introducción}

\subsection{Contextualización de la experiencia}

Esta innovación se desarrolló dentro del marco del PIME B-13 de la convocatoria 20152016.

La experiencia se ha realizado en la Escuela Técnica Superior de Informática (ETSINF) durante el citado curso académico, con dos tipos de alumnos:

Alumnos de la asignatura troncal Estadística de 6 créditos que se imparte en el primer curso del Grado en Ingeniería Informática (GII), y que se cursa en el segundo cuatrimestre. En concreto, fueron los de los grupos 1A, 1B y $1 \mathrm{H}$, con 117 alumnos matriculados de los que participaron 103.

Alumnos de la asignatura de 4,5 créditos obligatoria Técnicas de investigación e innovación del Máster Universitario de Gestión de la Información (MUGI), también del segundo cuatrimestre, con 20 alumnos matriculados, de los que participaron la totalidad.

\subsection{Justificación y motivación}

Los alumnos no suelen apreciar la utilidad de una asignatura de primer curso para su vida laboral. Sin embargo, cuando llega el momento de integrarse en un puesto de trabajo o de adquirir cierta formación de posgrado aplicada, necesitan poner en práctica estos conocimientos, pues son la base para comprender las complejas tareas de un profesional, especialmente en el campo de la ingeniería. Las razones por las que esto sucede son principalmente las siguientes: a) olvido: desfase temporal entre la impartición de esos contenidos y la necesidad de su aplicación; y b) incomprensión: el carácter generalmente más complejo y abstracto de estos contenidos (enfocados al uso del pensamiento abstracto, fundamental en las competencias de un ingeniero) provoca cierta indiferencia entre los alumnos a la hora de aplicarlos en casos reales.

Además, los actuales planes de estudio han llevado a que asignaturas que antes eran anuales hayan pasado a ser cuatrimestrales (con una considerable reducción del temario impartido), por lo que resulta complicado impartir los conocimientos necesarios para la titulación y poder explicar adicionalmente aplicaciones al mundo laboral.

La idea de utilizar un grupo de Facebook viene de la experimentación ya realizada en la UPV con los grupos de redes sociales (Calduch-Losa et al, 2014; Poza-Luján et al, 2014) y su valoración positiva, tanto por el alumnado como por el profesorado implicado. La utilización de estos entornos favorece entre los alumnos su participación en las actividades al sentirse en una plataforma que les permite desarrollar sus habilidades sociales tanto con los profesores como con el resto de compañeros. Así mismo, la plataforma permite de una forma sencilla utilizar diversas tecnologías para crear y difundir contenidos en distintos formatos que permitan la interactividad de todos los participantes, así como la medición del grado de participación y satisfacción.

(cc) EY-NC-ND 2018, Universitat Politècnica de València

Congreso IN-RED (2018) 


\section{Objetivos}

El objetivo general de este proyecto es que los alumnos mejoren la comprensión de las aplicaciones que la asignatura Estadística tiene en el mundo real, concretamente en el mundo laboral, para que puedan apreciar y captar mejor los contenidos de la asignatura, lo que redundará en la adquisición de nuevas habilidades que podrían ayudarles a mejorar su nivel profesional y laboral.

Los objetivos específicos que planteamos conseguir en el proyecto son los siguientes:

1. Identificar la percepción que los alumnos tienen de los contenidos de la asignatura Estadística.

Nivel de logro: Al iniciar y al finalizar el cuatrimestre se realizará una encuesta a los alumnos, y una de las cuestiones será que valoren la percepción que tienen acerca de la asignatura. Se contrastarán las respuestas que hayan dado en las dos ocasiones.

2. Incorporar el uso de las redes sociales en las asignaturas implicadas para fomentar la participación de los alumnos en el desarrollo del proceso enseñanza-aprendizaje.

Nivel de logro: La participación activa del grupo en la red social creado para el objeto del proyecto.

3. Fomentar la participación del alumnado, proponiendo preguntas directas después de cada ejemplo expuesto.

Nivel de logro: Que cada alumno del grupo responda y/o participe en los ejemplos expuestos.

4. Obtener interacción del alumnado de distintos perfiles.

Nivel de logro: La interacción se producirá, ya que en el mismo espacio de trabajo tendremos alumnos de grado y de máster, con la visión propia de la asignatura que estén cursando.

5. Trasladar los ejemplos a otros ámbitos.

Nivel de logro: Que los alumnos, a partir de los casos de estudio vistos, sean capaces de extrapolarlos o proyectarlos a otros entornos laborables de su interés.

6. Analizar estadísticamente las métricas web de las interacciones llevadas a cabo por los alumnos en la plataforma de Facebook, así como implicar a los alumnos en esta actividad con el fin de que éstos adquieran estas habilidades de análisis web.

Nivel de logro: Por una parte, conseguir obtener datos válidos para evaluar la actividad llevada a cabo y, por otra parte, que los alumnos sean capaces de participar en esta fase de análisis estadístico de métricas web.

\section{Desarrollo de la innovación}

Se creó un grupo cerrado de Facebook, en el que participaron los seis profesores implicados en el PIME y unos 120 alumnos. Los alumnos no estaban obligados a participar en él, pero 
Metodología para mejorar la percepción de utilidad de conceptos básicos estadísticos a través de Facebook

en el caso de la asignatura Estadística, su participación podía conllevarles el 10\% de la nota de la calificación final, que se obtiene participando en la asignatura, y que cada profesor puede elegir la manera de valorarla.

Los alumnos fueron comentando los artículos colgados en el grupo por el profesorado. De este modo se generó un debate, ya que los alumnos podían comentar sobre los artículos publicados y sobre las opiniones del resto de sus compañeros.

El primer trabajo compartido fue una ponencia presentada por algunos de los profesores en un congreso, en la que se hacía un estudio descriptivo de unos datos, y se les hacía preguntas a los alumnos, como puede verse en la Figura 1. Las preguntas se formulaban de una manera dirigida, para que los alumnos supieran las acciones que tenían que realizar.

\section{A. Ángeles Calduch Losa ha subido un archivo.}

Hola, aquí tenéis un caso práctico de Estadística Descriptiva. Comentadlo

e indicad de qué otro modo lo hubiérais explicado, qué pasaría si se aplicara a empresas de otros sectores, si los resultados son como esperábais, o si pensábais que habría más presencia online o en las redes sociales...

Fig.1 Ejemplo de pregunta a los alumnos

Iniciado el cuatrimestre, encontramos noticias online actuales en ese momento con matices estadísticos, por lo que redirigimos la orientación y la cantidad de los temas a tratar en el grupo, y finalmente se presentaron 10 posts en los que se combinaban casos prácticos y artículos divulgativos junto con artículos científicos con aplicaciones tanto de temas vistos en clase como de temas que no se ven, para que los alumnos vieran la utilidad de ir un poco más allá en la materia.

En la Figura 2 se ve el caso de un artículo científico redactado por algunos de los profesores participantes en el PIME, y en la redacción de la pregunta se intentó que los alumnos tuvieran que pensar en tipos de variables. Respecto a los métodos tratados, se combinaron uno visto en clase con uno que no, para que los estudiantes pudieran ver las similitudes y las diferencias entre ambos. 
Aquí está un estudio realizado con datos reales de los alumnos de la Escuela de hace algunos años. Los árboles de decisión no se ven en el temario de la asignatura Estadística, pero son intuitivos y creo que no tendréis ningún problema en interpretarlos; y regresión es el último tema de la asignatura.

Notad que tenemos variables tanto cualitativas como cuantitativas.

¿Por qué decimos que las técnicas utilizadas son predictivas?... Ver más

\section{Análisis del rendimiento académico en los estudios de informática de la Universidad Politécnica de Valencia aplicando técnicas de minería de datos.pdf}

Formato de documento portátil

Descargar Vista previa Subir nueva versión

\section{Me gusta \\ Comentar}

5

Ver 24 comentarios más

Este estudios debería realizarse en cualquier organización educativa porque muchas veces se aplican medidas sin saber realmente los efectos que tendrán en los estudiantes de nuevo ingreso en su primer año de carrera y son muy importantes para un buen s... Ver más Me gusta - Responder - 1

Este estudio al usar regresión te hace ver como todas las variables recogidas que en un primer contacto parecen independientes entre si pero con este metodo se analiza como dependen entre ellas, aunque con el sentido común la formación de los padres y... Ver más

Me gusta - Responder

Fig.2 Ejemplo de un artículo científico

Como se ha comentado con anterioridad, se incorporaron a la experiencia artículos divulgativos, extraídos de medios de comunicación online. En la Figura 3 tenemos un ejemplo de uno de ellos. 
Metodología para mejorar la percepción de utilidad de conceptos básicos estadísticos a través de Facebook

En la asignatura de Estadística hemos visto regresión, aunque no regresión logística, que es de la que trata este artículo.

Me gustaría que os informarais de lo que es la regresión logística y hablarais un poco de ella, así como de para qué otros casos la veis útil ¿Se os ocurre otra manera en la que se podrían pronosticar los resultados con los métodos vistos en clase?... Ver más

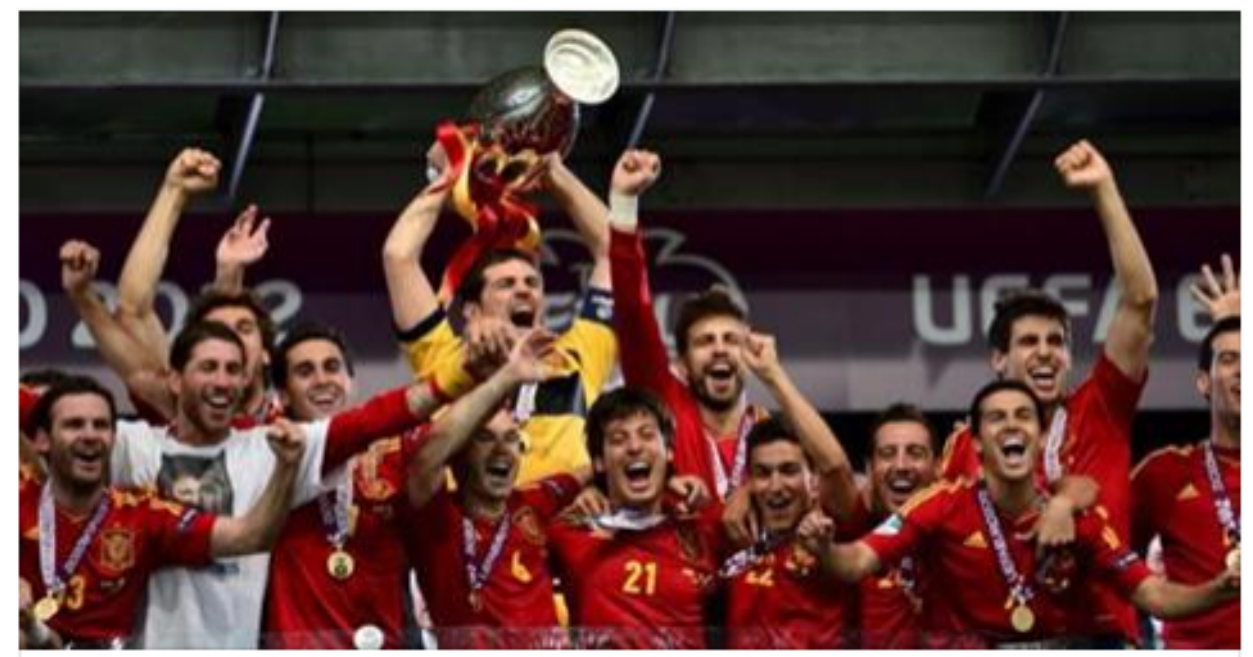

\section{Matemáticos predicen que el ganador de la Eurocopa será...}

Un grupo de matemáticos de la Universidad de Economía de Innsbruck ya sabe quién será el ganador de la Eurocopa 2016. Cuando todavía faltan 8 días para..

ELMUNDO.ES

It Me gusta $\quad \Rightarrow$ Comentar $\quad \Rightarrow$ Compartir

Ver 42 comentarios más

Fig.3 Ejemplo de un artículo divulgativo

Y en la Figura 4 puede apreciarse un caso práctico expuesto sobre métricas web, en concreto indicadores relativos al impacto de publicaciones científicas. 


\section{Enrique Orduña Malea}

En ocasiones, los Gobiernos, organizaciones o empresas se preguntan qué investigadores son aquellos que han logrado un mayor impacto. Quizás una posible forma de conocerlo es a través del impacto que han obtenido los trabajos de investigación que éstos han publicado durante su vida.

Pero... ¿qué ocurre cuando tengo muchos indicadores relativos al impacto?, ¿miden todos lo mismo?, ¿existen familias de indicadores que midan distintas dimensiones del impacto?

Quizás la realizaci... Ver más

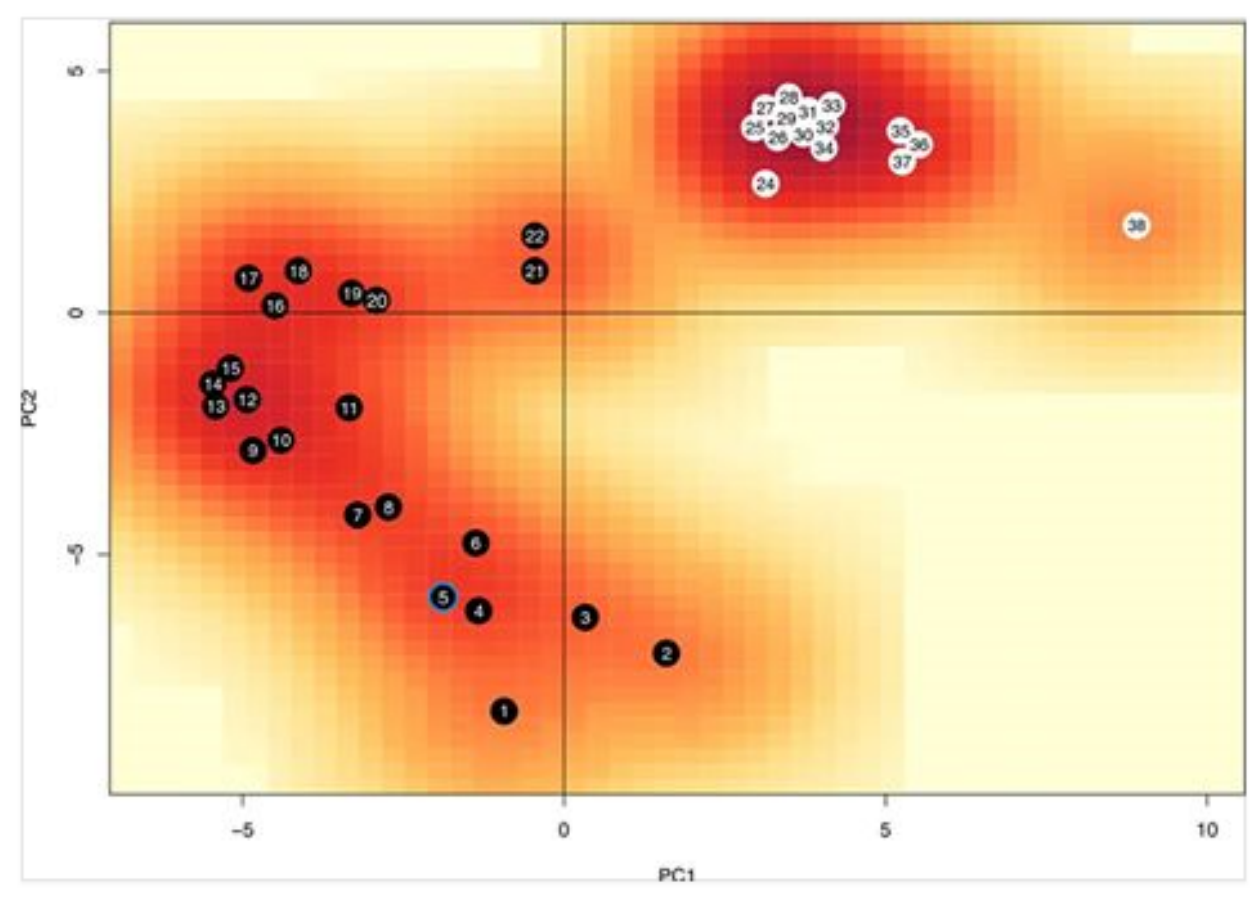

Me gusta

\section{Comentar}

Fig.4 Ejemplo de caso práctico sobre métricas web

\section{Resultados}

\section{1. ¿Se han conseguido los objetivos? ¿Qué resultados se han obtenido?}

Recordemos que el objetivo general de este proyecto es que los alumnos mejoren la comprensión de las aplicaciones que la asignatura Estadística tiene en el mundo real. Creemos que se ha cumplido con creces, por lo que nos han contestado en la encuesta final que se les ha pasado al acabar el cuatrimestre.

Veamos la consecución de los objetivos específicos: 
Metodología para mejorar la percepción de utilidad de conceptos básicos estadísticos a través de Facebook

1. Identificar la percepción que los alumnos tienen de los contenidos de la asignatura Estadística.

A los alumnos implicados se les han pasado dos encuestas: una al inicio del cuatrimestre, anónima mediante GoogleDocs, y otra al finalizar la asignatura, utilizando la plataforma Socrative. Por sus respuestas, la percepción de la asignatura ha mejorado.

2. Incorporar el uso de las redes sociales en las asignaturas implicadas para fomentar la participación de los alumnos en el desarrollo del proceso enseñanza-aprendizaje.

Han participado en el grupo de Facebook el $88 \%$ de los alumnos de grado y el $100 \%$ de los alumnos de máster.

3. Fomentar la participación del alumnado, proponiendo preguntas directas después de cada ejemplo expuesto.

Según los datos obtenidos por www.grytics.com, que es una herramienta que realiza estadísticas en los grupos de Facebook, el 89\% de las acciones realizadas en el grupo han sido comentarios, el 9\% corresponden a "me gusta", mientras que el 2\% restante son los posts que contenían los diversos artículos y trabajos presentados.

4. Obtener interacción del alumnado de distintos perfiles.

Aunque los alumnos de los dos niveles comentaron los artículos y trabajos presentados, las respuestas que se han dado entre ellos han sido entre compañeros de asignatura.

5. Trasladar los ejemplos a otros ámbitos.

En alguno de los ejemplos propuestos, ha habido alumnos que lo han replicado en otros campos, como puede verse en la Figura 5, ya que han realizado sorteos con compañeros de clase o con deportistas.

6. Analizar estadísticamente las métricas web de las interacciones llevadas a cabo por los alumnos en la plataforma de Facebook, así como implicar a los alumnos en esta actividad con el fin de que éstos adquieran estas habilidades de análisis web.

Se ha medido la participación del alumnado en el grupo, pero no se les ha hecho partícipes de ello.

(cc) EY-NC-ND 2018, Universitat Politècnica de València

Congreso IN-RED (2018) 
Como dice en el artículo, una solución sencilla seria asignar un número a cada alumno, con esto conseguiríamos que cada alumno que quiere entrar tenga la misma probabilidad de ello. Para demostrarlo he creado un pequeño programa en java que genera números aleatorios (entre 1 y el número de alumnos que quieren entrar) y va sumando el número de veces que sale. Yo he cogido como ejemplo la lista de la clase $1 \mathrm{H} 2$ y les he asignado un número (en total son 20 alumnos). He generado posteriormente 1000 números aleatorios y he conseguido los siguientes resultados:

Cada alumno tiene un numero asignado seguido del número de veces que ha salido y su porcentaje de aparición. (Ver imagen)

Como podemos observar el número que más ha salido ha sido el de Ismael (el 9). Si volviésemos a ejecutar el programa estos resultados cambiarían totalmente. Pero en definitiva todos tienen la misma probabilidad de entrar (1/20), ya que como se puede apreciar están muy ajustados los resultados. Además, podemos generar más números aleatorios para evitar posibles empates.

Comparto el código del programa:

https://gist.github.com/.../d23a7c2d1e0b262d0f14f89fcb14f023

\begin{tabular}{|c|c|c|c|}
\hline & Alfonso Safont, Adrian & 45 & $4,5 e s$ \\
\hline & Almela Seller, Carlos & 44 & 4,405 \\
\hline & Anchel Urda, David & 51 & $5,1 e x$ \\
\hline 4 & Ávila Fernández, Sergi & 58 & $5,8 e x$ \\
\hline 5 & Ben Kasem Agbalu, Isaac & 53 & $5,3 e x$ \\
\hline & Cerro Montoya, Pablo & 53 & $5,30 x$ \\
\hline & Correa Julve, Jorge & 41 & $4,1 e x$ \\
\hline & Costa Vidal, Victor & 54 & $5,4 e x$ \\
\hline & Diaz Segrelíes, Ismael & 64 & 6,408 \\
\hline 10 & Dolz Martinez, Vicent & 53 & $5,3 e x$ \\
\hline 11 & Fernández De La Torre, Diego & 59 & 5, gex \\
\hline 12 & Gavila Martinez, Joan & 58 & $5,8 e s$ \\
\hline 13 & López Andrango, Kevin Danilo & 39 & $3,9 e x$ \\
\hline 14 & Martinez Ponce, Raúl & 46 & $4,6 e s$ \\
\hline 15 & Navarro Font, Cristina & 51 & $5,10 x$ \\
\hline 16 & Palomares Badenas, Raúl & 42 & 4,205 \\
\hline 17 & Pérez Martinez, Alejandro & 49 & $4,9 e x$ \\
\hline 18 & Rivas Ferri, Francísco José & 43 & 4,305 \\
\hline 19 & Sahuquillo Soriano, Adrian & 5) & 5,705 \\
\hline 20 & Sinues Tosca, Juan Jose & 40 & 4, ees \\
\hline
\end{tabular}

Me gusta - Responder - 17

Realizando un Sorteo por Letra similar al realizado en el artículo de los jugadores del Valencia Basket, he obtenido los siguientes datos de estudio:

\begin{tabular}{|l|lllr}
\hline 1 & Apellido & Nombre & Probabilidad Porcentaje \\
2 & DIOT & Antoine & $216 / 729$ & 29 \\
\hline
\end{tabular}

Fig.5 Ejemplo de réplicas por parte de los alumnos

\subsection{Metodología y técnicas utilizadas para el tratamiento y análisis de los datos}

Antes de comenzar con la experiencia, se consultó a los alumnos sus opiniones iniciales acerca de la asignatura a través de una encuesta realizada en GoogleDocs. Estos datos fueron vistos mediante Excel y el paquete estadístico Statgraphics Centurion XVI, para saber cuál era el punto de partida.

A partir de ahí, los temas tratados en el grupo de Facebook se colgaron como posts mediante documentos pdf o enlaces a medios digitales. Los datos de participación en el grupo han sido tomados y medidos con la herramienta Grytics (www.grytics.com). Paralelamente, se confeccionó en Excel un listado con las participaciones de cada uno de 
los alumnos de la asignatura Estadística, para poder puntuarlas como nota de clase. Al finalizar la asignatura se han obtenido las opiniones del alumnado con la herramienta Socrative (www.socrative.com). Esta app proporciona los datos en Excel, y de nuevo han sido analizados con el paquete estadístico Statgraphics Centurion XVI.

\section{Conclusiones}

La experiencia ha aunado aplicaciones de nuestras asignaturas con las redes sociales (en este caso Facebook), y se ha podido comprobar un alto grado de aceptación por parte de los alumnos. A los estudiantes les ha gustado trabajar con ejemplos reales, lo que nos ha permitido mejorar su grado de percepción de utilidad de la materia.

Mediante la aplicación combinada de plataformas de redes sociales y de herramientas para obtener métricas de uso, resulta relativamente sencillo replicar experimentos similares, que podrían ser extrapolables a otras asignaturas que adolezcan de similares problemas (escasa percepción de utilidad por parte de los estudiantes de ingeniería y materias fundamentadas en conceptos abstractos).

Tras la experiencia, se ha seguido trabajando de forma satisfactoria con los grupos cerrados de Facebook en alguna de nuestras asignaturas.

\section{Referencias}

CALDUCH LOSA, Á., POZA-LUJÁN, J.L., TERUEL, D., ALBORS SOROLLA, A., CABRERA, M., REBOLLO, M. (2014). "Pautas de actuación para optimizer el uso de las redes socials en la docencia universitaria”. En Jornadas de Enseñanza Universitaria de la Informática (JENUI). Oviedo. Actas de las XX Jenui. 59 - 65

POZA-LUJÁN, J.L., CALDUCH-LOSA, Á., ALBORS, A., CABRERA, M., TERUEL, D., REBOLLO, M. y DÍEZ, R. (2014). "Propuesta de parámetros y caracterización de los grupos de las redes sociales orientados a la docencia universitaria: experiencia y resultados" en Revista de Educación a Distancia (RED). Año XIII. Número 44. (http://www.um.es/ead/red/44)

CAMACHO MARTÍ, M. (2010). "Las redes sociales para enseñar y aprender. Reflexiones pedagógicas básicas” en Castañeda Quintero, L. Aprendizaje con redes sociales. Tejidos educativos para los nuevos entornos. Sevilla: Eduforma. 91 - 104

SANMARTÍN, O.R. (2016). "Tres de cada 10 universitarios no ven útil su título para encontrar trabajo”. En El http://www.elmundo.es/sociedad/2016/07/22/57911b17e2704ec2158b464f.html [Consulta: 23 de julio de 2016]

TÚÑEZ LÓPEZ, M., SIXTO GARCÍA, J. (2012). "Las redes sociales como entorno docente: análisis del uso de Facebook en la docencia universitaria” en Pixel-Bit.Revista de Medios y Educación. Número 41. 77 - 92

(cc) EY-NC-ND 2018, Universitat Politècnica de València 